\title{
NEW ADAPTIVE REBROADCASTING USING NEIGHBOUR LEARNING FOR WIRELESS NANOSENSOR NETWORKS
}

\author{
Ayoub Oukhatar ${ }^{1)}$, Driss El Ouadghiri 1), Mohamed Bakhouya ${ }^{2)}$ \\ 1) Laboratory of Informatics and Applications, Faculty of sciences, Moulay Ismail University, Meknes, Morocco \\ ${ }^{2)}$ International University of Rabat, Morocco Technopolis Rabat-Shore, 11100 Sala el Jadida, Morocco
}

Paper history:

Received 3 June 2020

Received in revised form 9 September 2020

Accepted 23 November 2020

Available online 30 December 2020

Keywords:

Wireless Nanosensors Networks;

Nanonetworks;

Nanocommunication;

Protocols;

Routing.

\begin{abstract}
Wirelesse Nanosensor Networks (WNSNs) contain a large number of independent and mobile nanodevices assembled with nanotransceivers and nanoantennas to work in Terahertz frequency band $(0.1-10 \mathrm{THz})$. These nanodevices exploit the properties of modern nanomaterials to recognize new varieties of events at the nanoscale, such as the presence of harmful viruses or bacteria and the detection of low concentrations of chemical and harmful gas molecules. Communication between nanonodes can be established by using molecular or electromagnetic communication approaches. One of the major problems of wireless nanosensor networks is the limited resources of nanodevices (e.g., computation, memory and power). On the other hand, such limited capacity cannot simply ensure communication between nanonodes using the flooding mechanism, which affects network performance and increases resource utilization. This paper considers the electromagnetic-based wireless nanosensor networks, and proposes a New Adaptive Probabilistic Based Broadcast Using Neighborhood Information. Simulations have been conducted using Nanosim simulator in order to compare our new schemes with the fixed probabilistic based broadcast. The experiments show that the proposed approach gives good results in terms of Packet Delivery Ratio (PDR reached 95\%.), the amount of energy consumed (significantly reduced) for all the categories of density. No startup setup is required: the nanonodes adjust by themselves the broadcasting probability based on neighborhood collected information.
\end{abstract}

Copyright (C) Research Institute for Intelligent Computer Systems, 2020. All rights reserved.

\section{INTRODUCTION}

The wireless nanosensor network has gained increasing attention over the past two decades due to the requirement for discovering and measuring chemical and physical phenomena in difficult-toreach biological, industrial and environmental systems that are within the nanoscale level. With continued advances in nanotechnology tools and a growing understanding of nanoscale phenomena, recent developments in nanotechnology engineering are leading to the approach of nano-machines creation. Nano-machines are sensing devices with at least one of their sensing dimensions being no greater than one to hundred nanometers. These are the most elementary significant units in the nanoworld that can perform very simple tasks, detection and actuation [4]. The WNSN is generally composed of several nanosensor nodes that are autonomous, tiny, low-cost and low-energy. These nanonodes collect data from their environment and collaborate to transmit the detected data to centralized processing units called nanointerfaces for further processing [3]. The communication capability will allow the nano-machine to operate synchronously, supervised and cooperatively to achieve a common goal. This will greatly increase the capacities and application areas of nano-machines. Due to the small size of the nano-machines, their working space is very limited and, for this reason, in a small operational area of a few meters, hundreds of nanomachines will be diffused arbitrarily. Deploying a dense and interconnected nano-machine will increment the capabilities of a single nano-machine. The small size of the nanodevice will make it troublesome to control them, while interconnected 
nano-machines can be controlled using broadcasting and multihop interconnection procedures. Therefore, data dissemination mechanisms are required to allow these nanodevices to exchange captured data with each other and with nanointerface units. Flooding is traditionally used for broadcasting in electromagnetic-based WNSNs. This is very simple and requires few resources in the nanonodes but generates a significant overload on the network [12].

This paper presents New Adaptive Probabilistic Based Broadcast Using Neighborhood Information for electromagnetic-based communication WNSNs in order to decrease the power consumption and increase the data transmission success rate. The protocol is well adapted for different density ad-hoc networks, with frequent changes in neighbors list. Firstly, the decision for rebroadcasting is localized and based on information collected periodically from the nanonode environment. Secondly, a nanonode sends a packet without imposing its own broadcasting probability to its neighbors. The proposed broadcasting protocol is compared with different fixed probability methods. Simulations are established to study the performance and the effectiveness of the proposed approach. The remainder of this paper is organized as follows. Section II provides an overview of WNSN and related works. The adaptive probabilistic algorithm is presented in Section III. Simulation results analysis is demonstrated in Section IV. Section V presents the arranged work and concludes the document.

\section{BACKGROUND AND RELATED WORK}

In this section, an EM-WNSNs overview and the work related to existing information dissemination mechanisms in wirelless nanonetworks are presented.

\subsection{WNSNs BACKGROUND}

The EM-WNSNs are defined as the Interconnection of nanoscale devices with conventional network system (i.e., WiFi, cellular networks, and so on) and vice-versa [4]. Wireless nanosensor network (WNSN) infrastructure can be deployed by mixing nanodevices and several other existing communication networks like IoT, Sensors Network, Cloud Computing, Data science analyses, etc. As presented in [4] a generic WNSN should be composed of three categories of nanodevices: Nanonodes are small and simple devices with very restrained energy, computational, and storage capabilities. They can be diffused into a target area for capturing and providing information about the neighborhood. Nanorouters are nanomachines having wider sizes and assets than a simple nanonode. They are engaged in controlling the behavior of nanonodes by involving short management messages. Nanointerface is most convoluted devices able to act as a gateway between the nano and the micro scale world.

Nanosensors are nanoscale devices or peripherals that are capable of achieving the simple tasks of computation, sensing, and actuation at nanoscale level, they have a restrained size and limited complexity, for this reason, WNSN investigated novel nanomaterial properties such as CNT (carbon nanotube) and GNR (graphene nanoribbon) will permit to form nanomaterial based antennas which can work within the Terahertz Band, within 0.1 and $10 \mathrm{THz}$ [11], while having a form factor appropriate to be integrated into nanosensors. Moreover, exceptionally high propagation losses and a very large accessible bandwidth are the most Terahertz Band communication characteristics [9] which need to be taken under consideration to create effective WNSNs models and protocols, while jointly taking into account the limited functioning, sensing, actuating and computing. The peculiarities of the terahertz band blended with the drawbacks of the nano-machines call for a simple communication approach. In [10], a new communication scheme called Time Spread On-Off Keying (TS-OOK) is introduced. TS-OOK makes use of very quick pulses inside the range of femtoseconds where each logical pulse "1" is transmitted by the use of a Femto second lengthy pulse and a logical " 0 " is transmitted as silence. This scheme helps to decrease the impact of atomic absorption noise which results in reduction of energy utilization.

\subsection{RELATED WORKS}

As mentioned before, nanomachines are characterized by constrained memory, computing and energy capacity. Consequently, the conventional routing model and corresponding routing mechanism are not appropriate for WNSN. Flooding may be considered as the routing protocol that will operate in WNSN. This is typically one of the simplest routing techniques. In this technique, each nanonode in the network transmits the flooding message. However, simple broadcast by flooding is generally costly and outcomes in intense redundancy and collisions in the network, this type of state has regularly been referred to as a broadcast storm problem [20]. To solve the broadcast storm issue, a series of threshold-based broadcasting schemes had been proposed [17]. Below, we review two representative schemes: the parameter-based scheme which depends upon certain threshold (e.g., distance, redundant message counts, or broadcast probability) values to estimate the network density in $[15,16]$. 
The area-based scheme uses geographical region of transmitter to choose a further transmission range. In this approach, only a neighbor, which is positioned farthest from a transmitter, rebroadcasts the message in the distance-based scheme.

The forwarding approaches in traditional WSNs do not consider nanoscale properties of nanodevices (limited memory and processing capabilities). Compared to molecular communications [5], and the existing research on information dissemination techniques focused on energy conservation. In Table 1, we have compared each existing information dissemination protocols according to several factors, including the deployment space, the network topology, routing path, computing capacity, nanonode mobility, position awareness, energy- aware and storage requirements. The conception and implementation of routing protocols are considered imperative in WNSN. This is because of nanonetwork of nanodevices is usually constrained in their processing capacity, communication range and energy capacity. In any case, several design perspectives should be taken into consideration such as the nanonetwork topology, deployment space, nanonode mobility, and energy consumption. In fact, energy is the foremost critical and restricting factor in any areas of monitoring application by using WNSN. In that sense, routing protocols which optimize the energy consumption while satisfying diverse constraints are expected to have an incredible impact on the WNSN paradigm.

Table 1. Comparison of WNSNs routing protocols.

\begin{tabular}{|c|c|c|c|c|c|c|c|c|c|c|}
\hline $\begin{array}{l}\text { Routing } \\
\text { Protocols }\end{array}$ & RADAR & CORONA & DEROUS & SLR & MHTD & EEMR & ECR & TTLF & RDDA & PBDA \\
\hline References & [22] & [19] & [13] & [18] & [24] & [8] & [14] & {$[21]$} & [2] & [23] \\
\hline $\begin{array}{c}\text { Deployment } \\
\text { space }\end{array}$ & $2 \mathrm{D}$ & $2 \mathrm{D}$ & $2 \mathrm{D}$ & $3 \mathrm{D}$ & $2 \mathrm{D}$ & $2 \mathrm{D}$ & $3 \mathrm{D}$ & $2 \mathrm{D}$ & $2 \mathrm{D}$ & $2 \mathrm{D}$ \\
\hline $\begin{array}{l}\text { Network } \\
\text { topology }\end{array}$ & Flat & Tree & Flat & Flat & Tree & Tree & Tree & Flat & Tree & Tree \\
\hline $\begin{array}{c}\text { Routing } \\
\text { Path }\end{array}$ & $\begin{array}{l}\text { Multi } \\
\text { Path }\end{array}$ & $\begin{array}{l}\text { Multi } \\
\text { Path }\end{array}$ & $\begin{array}{l}\text { Multi } \\
\text { Path }\end{array}$ & $\begin{array}{l}\text { Multi } \\
\text { Path }\end{array}$ & $\begin{array}{l}\text { Single } \\
\text { Path }\end{array}$ & $\begin{array}{l}\text { Single } \\
\text { Path }\end{array}$ & $\begin{array}{l}\text { Single } \\
\text { Path }\end{array}$ & $\begin{array}{l}\text { Single } \\
\text { Path }\end{array}$ & $\begin{array}{c}\text { Single } \\
\text { Path }\end{array}$ & $\begin{array}{l}\text { Single } \\
\text { Path }\end{array}$ \\
\hline computing & Low & Low & Medium & Medium & High & High & Medium & Low & High & High \\
\hline Mobility & static & static & static & static & static & static & static & static & static & static \\
\hline $\begin{array}{c}\text { Energy- } \\
\text { aware }\end{array}$ & No & No & No & No & Yes & Yes & Yes & Yes & Yes & Yes \\
\hline $\begin{array}{c}\text { Storage } \\
\text { requirements }\end{array}$ & Low & Low & Medium & High & High & High & Low & Low & Low & Medium \\
\hline $\begin{array}{c}\text { Position } \\
\text { awareness }\end{array}$ & No & $\begin{array}{c}\text { Hop } \\
\text { counts to } \\
\text { the anchor } \\
\text { nodes }\end{array}$ & $\begin{array}{l}\text { Hop } \\
\text { counts to } \\
\text { raduis }\end{array}$ & $\begin{array}{c}\text { Hop } \\
\text { counts } \\
\text { to the } \\
\text { coordi- } \\
\text { nate } \\
\text { node }\end{array}$ & $\begin{array}{c}\text { Distance } \\
\text { to the } \\
\text { nano } \\
\text { controller }\end{array}$ & $\begin{array}{c}\text { Distance } \\
\text { to the } \\
\text { nano } \\
\text { controller }\end{array}$ & Layer & No & $\begin{array}{c}\text { Hop } \\
\text { counts } \\
\text { to } \\
\text { center } \\
\text { node }\end{array}$ & $\begin{array}{c}\text { Hop counts } \\
\text { to } \\
\text { ClusterHead }\end{array}$ \\
\hline
\end{tabular}

In this work, we first investigated fundamental data forwarding approaches to WSN as starting points for designing forwarding algorithm for EMWNSNs, secondly we propose a New Adaptive Probabilistic Based Broadcast Using Neighborhood Information of nanosensors with the aim to minimize the energy consumption, improve the data delivery and ameliorate the latency in WNSNs.

\section{THE ADAPTIVE PROBABILISTIC BASED BROADCAST ALGORITHM}

In this section we have adapted probabilisticbased broadcast approach using neighborhood information in WNSNs. Before describing the procedure of this mechanism, certain specifications need to be presented: $\mathbf{P}_{\mathbf{c}}$ presents the probability threshold of rebroadcasting a packet, this parameter will be utilized for selecting the rebroadcast strategy, 
each nanonode has its own broadcast probability indicated by $\mathbf{p}, \mathbf{p}$ will be set up arbitrarily between 0 and 1 , at the same time the probability $\mathbf{P}_{\mathbf{c}}$ is computed from the regional density (i.e., the number of neighbors $\mathbf{N}_{\mathbf{g h}}$ ) and the number of times the broadcast packet is received $\mathbf{C}_{\mathbf{m}}$. As Algorithm 1 states, Each nanonode will calculate its broadcasting probability $\mathbf{P}_{\mathbf{c}}$ threshold as bellow:

$$
P_{c}=\frac{N_{g h}-C_{m}}{N_{g h}}
$$

Each nanonode has such a file for saving packet information and includes unique ID of a packet and Boolean variable $\mathbf{b}$ for proving in case the progressing packet was already rebroadcasted by the nanonode. The $\mathbf{P}_{\mathbf{c}}$ equation was chosen because, normally, the probability of diffusion is inversely proportional to the density of neighborhood. In addition, the global number of neighbors for each nanonode is denoted by $\mathbf{N}_{\mathrm{gh}}$. The term $\mathbf{C}_{\mathbf{m}}$ provides an idea on the neighbor that has already received the packet. While, the $\mathbf{N}_{\mathbf{g h}}-\mathbf{C}_{\mathbf{m}}$ terms present the number of neighbors of a given nanonode that has not received the packet Fig. 1, and therefore if this number is high and the node participates positively in the transmission of packet, it must rebroadcast this packet to its neighbors (broadcasting probability threshold needs to be high). However, if this number is low, the nanonode should not rebroadcast the packet (broadcasting probability threshold needs to be low) Fig. 2.

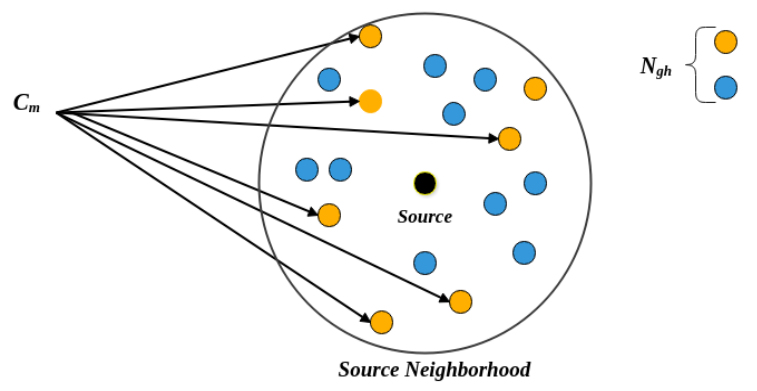

Figure 1 - An example of a Nanonode environment

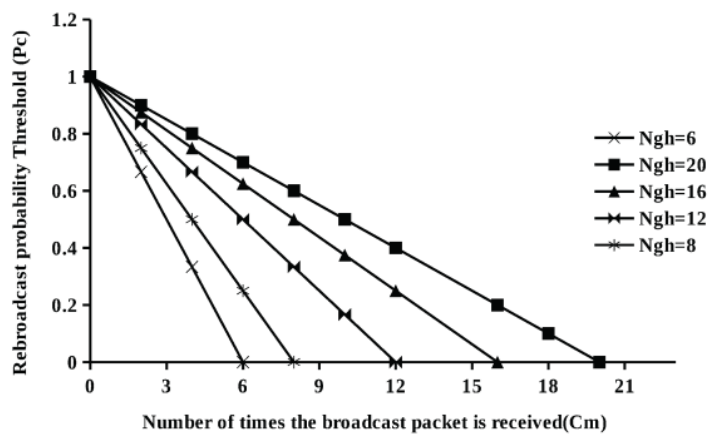

Figure 2 - Optimal forwarding probability according to $95 \%$ PDR
Alghorithm 1: Adaptive probabilistic algorithm.

Input: Number of Duplicated message $\mathbf{C}_{\mathbf{m}}$, and node $\mathbf{X}$ 's neighbors'number $\mathbf{N}_{\mathbf{g h}}$.

Output: The broadcasting probability Threshold $\mathbf{P}_{\mathbf{c}}$ node $\mathbf{X}$ gets assigned.

1. On receiving a sending packet $\mathbf{m}$ at nanonode $\mathbf{X}$;

2. Score the attribute ID from the packet and the b value;

3. Score the scale $\mathbf{N}_{\mathrm{gh}}$ of nanonode $\mathbf{X}$ (sum of neighbors of nanonode $\mathbf{X}$ );

4. Score the totale of times the packet is received $\mathbf{C}_{\mathbf{m}}$;

5. Adjusting The broadcasting probability Threshold $\mathbf{P}_{\mathbf{c}}$ based on $\mathrm{N}$ gh and $\mathbf{C}_{\mathbf{m}}$;

6. Set up an arbitrary probability $\mathbf{p}$ among $[0,1]$

if $\left(\mathbf{p}<\mathbf{P}_{\mathbf{c}}\right)$

then

rebroadcast the packet ;

$\mathbf{b}=$ TRUE;

else

drop it;

end

The algorithm enables a nanonode to learn by interacting with its surrounding environment. The nanonode will learn to calculate its broadcasting probability threshold that can maximize its longterm rewards by using its own experience. As shown in Fig. 3, a nanonode regularly updates its probability threshold based on the achieved inputs. The advantage of this model is that the braodcasting probability threshold value is not the same for all network nanonodes, so this value will be adjusted to each nanonode according to its environment, another advantage is that this scheme does not require any addition of information in the packet, Therefore no energy consumption will be added.

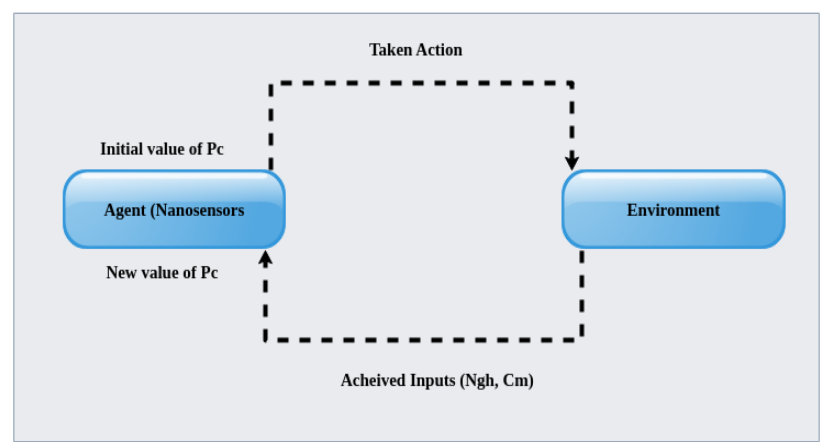

Figure 3 - A Visualization of the $P_{c}$ determination 


\section{PERFORMANCE EVALUATION}

To evaluate the performance of the proposed approach, Nano-Sim is proposed as a powerful tool to modeling electromagnetic based nanonetworks [6]. It is developed in $\mathrm{C}++$ and integrated as a submodel of NS-3 which is a discrete-event network simulator. It is free software, and is freely accessible for research [1]. In order to assess and study the performance of the proposed approach, a various scenarios have been simulated using the Nano-sim simulator. In this work we assess our approach in terms of tree main performance metrics:

- Packet delivery ratio (PDR): This indicates the percentages of packets delivered within a specified period. PDR is defined as the ratio of real packet delivered to full packets sent. This is the successful delivery rate of messages on a communication channel.

- Energy consumption: according to the energy model given in [9], we focus on the power consumed at the time of the transmission and reception of packets. Moreover, the energy required to accomplish the reception and the transmission of a packet of $\mathrm{N}$ bits can be given by:

$$
\begin{gathered}
E_{\text {packet }}^{t x}=N_{\text {bits }} * L * E_{\text {pulse }}^{t x} \\
E_{\text {packet }}^{r x}=N_{\text {bits }} * E_{\text {pulse }}^{r x}
\end{gathered}
$$

where $E_{\text {pulse }}^{\text {tx }}=1 P J$ and $E_{\text {pulse }}^{r x}=0.1 P J$ are, respectively, the energy required at time of transmission and reception of one pulse, and $\mathbf{L}$ indicates the probability of getting a 1 bit within the packet of $\mathbf{N}$ bits (typically, Li equals to $\mathbf{0 . 5}$ because the symbols are equiprobable).

- Average Delay: it demonstrates how much time it takes for a packet to get from one assigned nanonode to the nanointerface.

As the first step to asses the performance of our proposed approach, we present our system model here. This system includes a simplified model as the architecture for our special WNSNs application. We have assumed that nanonodes are set in cuboid region and they move according to the GaussMarkov mobility model. At the beginning of the simulation, the nanonodes are consistently diffused interior target zone for capturing data from its surrounding environment. Nanonodes move taking after one direction arbitrarily chosen at the speed of $30 \mathrm{~cm} / \mathrm{s}$. The nanointerface is deployed at the center of the cuboid and keeps its location fixed during the simulation time. Each nanonode my change its position during simulation time according to the mobility model. Nanonodes collect data about surrounding environment and irregularly broadcast it to its neighbors. As a result, packets are diffused from one nanonode to other intermediate ones until they achieve the nanointerface which can acts as an intermediate device between the WNSN and the making decision unit Fig. 4.

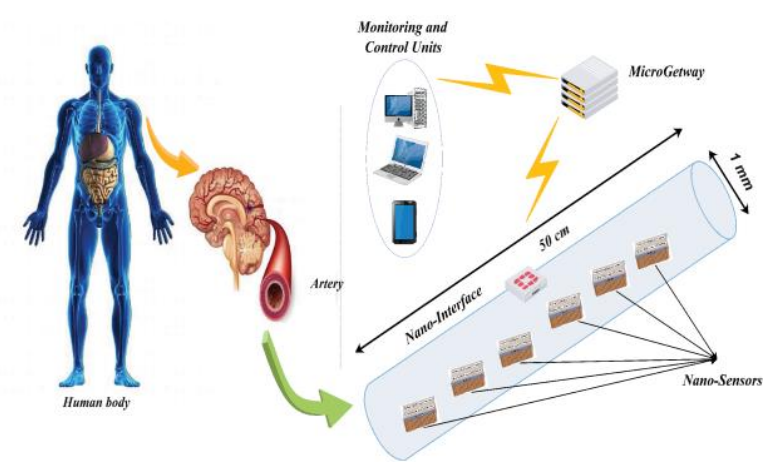

Figure 4 - A proposed model system for nanosensor network

\subsection{SIMULATION PARAMETERS}

The employed simulation configuration has been chosen in accordance with [7] and is presented in Table 2. In order to evaluate nanonetwork in context of average latency, PDR and the energy consumed in the transmission and reception of data, several simulations scenarios for WNSN are conducted as presented in Table 3 .

Table 2. The parameters used in the simulation

\begin{tabular}{|l|c|}
\hline \multicolumn{1}{|c|}{ Parameters } & Values \\
\hline Simulation-duration & $1 \mathrm{~s}$ \\
\hline Density of nanonodes & from 100 to 500 \\
\hline Pulse_energy & $100 \mathrm{pJ}$ \\
\hline Pulse_duration & $100 \mathrm{fs}$ \\
\hline Pulse_inter-arrival time & $10 \mathrm{ps}$ \\
\hline Packet_size & 128 bytes \\
\hline Generation_packet_interval & $0.5 \mathrm{~s}$ \\
\hline Area & $10^{-3} * 10^{-3} * 0.5 \mathrm{~m} \mathrm{3}$ \\
\hline
\end{tabular}

Table 3. Variation of network parameters

\begin{tabular}{|l|l|c|}
\hline 1 & \multicolumn{1}{|c|}{ Fixed } & \multicolumn{1}{c|}{ Varied } \\
\hline 1 & $\begin{array}{l}\text { Protocol=simple-flooding } \\
\text { Tr_range }=0.01 . \\
\text { Generation_packet_interval }=0.5 \mathrm{~s} .\end{array}$ & $\begin{array}{c}\text { Number of nodes } \\
\text { from } 100 \text { to } 300 \\
\text { with step 50. }\end{array}$ \\
\hline 2 & $\begin{array}{l}\text { Protocol=probabilistic-based } \\
\text { Tr_range }=0.01 . \\
\text { Generation_packet_interval }=0.5 \mathrm{~s} .\end{array}$ & $\begin{array}{c}\text { Number of nodes } \\
\text { from } 100 \text { to } 300 \\
\text { with step 50 }\end{array}$ \\
\hline 3 & $\begin{array}{l}\text { Protocol=Adaptive-algorithm } \\
\text { Tr_range=0.01. } \\
\text { Generation_packet_interval }=0.5 \mathrm{~s} .\end{array}$ & $\begin{array}{l}\text { Number of nodes } \\
\text { from } 100 \text { to } 300 \\
\text { with step 50. } \\
\text { Threshold values } \\
\text { (P) from } 0.2 \text { to } 1 \\
\text { with step } 0.2\end{array}$ \\
\hline
\end{tabular}




\subsection{SIMULATION RESULTS}

In the first scenario of simulations, we have examined network behavior by varying the probability threshold value of rebroadcasting a received message $\boldsymbol{P}$. This parameter will be utilized for selecting the rebroadcast strategy, each nanonode has its own broadcast probability indicated by $\mathbf{p}, \mathbf{p}$ will be set up arbitrarily between 0 and 1 , if $\mathbf{p}<\mathbf{P}$ the nanonode rebroadcasts the messages, other messages will be dropped. Theoretically, it is anticipated that via reducing this threshold value, rebroadcast messages must certainly be reduced, but a decrease in the threshold could result in a low PDR value. Therefore, the threshold must be adjusted according to the network density. Flooding and the fixed probabilistic-based Broadcast schemes are compared in figures below, simulations are realized by fixing the transmission range $(0.01)$ and generation packet interval (0.5). Number of nanonodes changed from 100 to 300 . Fig. 5 compares the two schemes in context of varying threshold. We can see that the average delay can be distinct for various thresholds. This is the result of the irregular generation of a brief time interval. The received packets were diffused after the time slot.

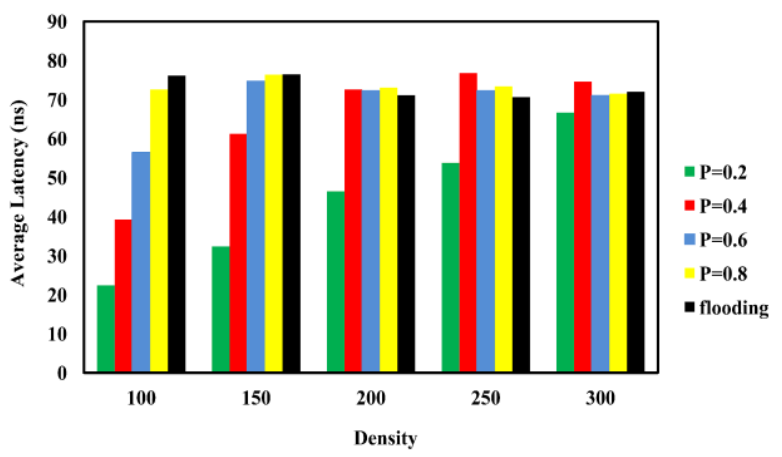

Figure 5 - Impact of Density: average delay.

Fig. 6 illustrates that, when the network is sparse, low threshold value generates a low packet delivery rate, e.g., the PDR reached $23 \%$ in average when the number of nanonodes is equal to 100 and the threshold is fixed to 0.2. While, in the case of the dense network, good results of PDR are achieved with the same threshold values used, for example, the packet delivery ratio attains $95 \%$ when the number of nanonodes is equal to 300 and the threshold is fixed to 0.2 .

Small threshold value of dissemination probability gives good results for the energy utilization (see Fig. 7). By extending threshold, number of transmissions and receptions tends towards the results obtained in the case of flooding scheme. We checked that when threshold value is close to 1 , this factor will be the same as within the case of basic flooding. Following the analysis of these figures and by considering the number of nanonodes in the network, the experiments demonstrate that by setting the probability threshold at 0,6 , in dense topology, satisfactory values for all performance evaluation metrics are obtained, i.e., PDR reaches nearly 98\%, the energy consumption is reduced, and good results are expected for average delay, whereas this threshold value gives weak performance in sparse topology. Therefore, the adaptation of the rebroadcast probability threshold value to the network density must be used.

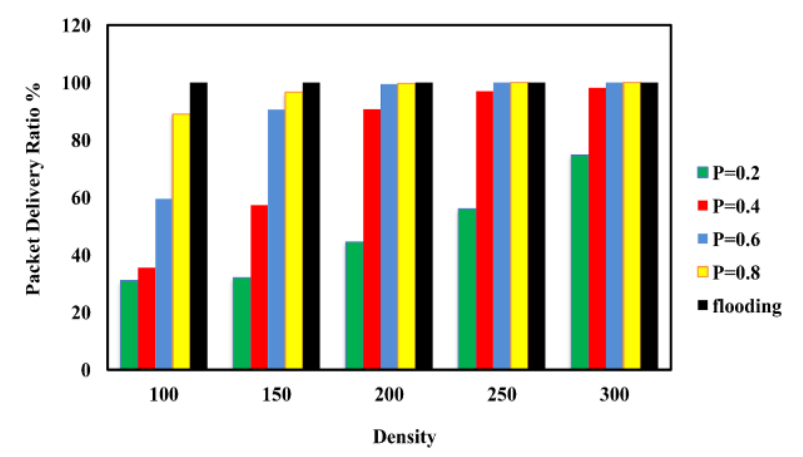

Figure 6 - Impact of Density: PDR.

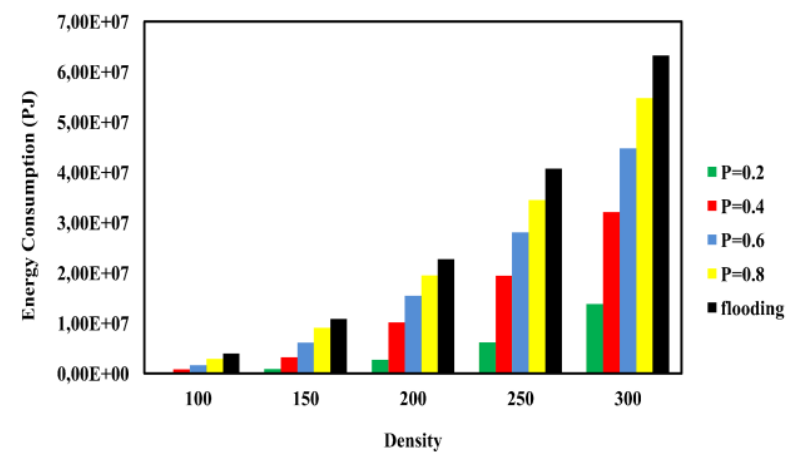

Figure 7 - Impact of threshold: Energy Consumption

In the second scenario, we have compared our new adaptive probabilistic-based broadcast scheme with fixed probabilistic based one by changing the number of nanonodes in the networks and checking out the behaviors of the Packet Delivery Ratio, the Energy Consumption and the average latency for each different probabilistic broadcast threshold.

Fig. 8 presents the performance of the adaptive based broadcast using neighborhood learning in terms of Packet Delivery Ratio. The results show that the protocol gives good results in terms of PDR (PDR reached 95\%.) for all the categories of density. No startup setup is required: the nanonodes adjust by themselves the probability threshold $\mathbf{P}_{\mathbf{c}}$ based on environment information's which is essential for a great rebroadcasting. Furthermore, the expression $\mathbf{N}_{\mathbf{g h}}-\mathbf{C}_{\mathbf{m}}$ (used in the formula (1)) is exceptionally valuable for partial broadcast. It gives information 
about the neighbors that have not received the message in the nanonode environment. If this parameter is high and in order to participates positively in the transmission of message, the nanonode has to broadcast the packet to its neighbors. On the other hand if this parameter is low (i.e., the majority of neighbors have already received the same packet) the nanonode has to delete the packet.

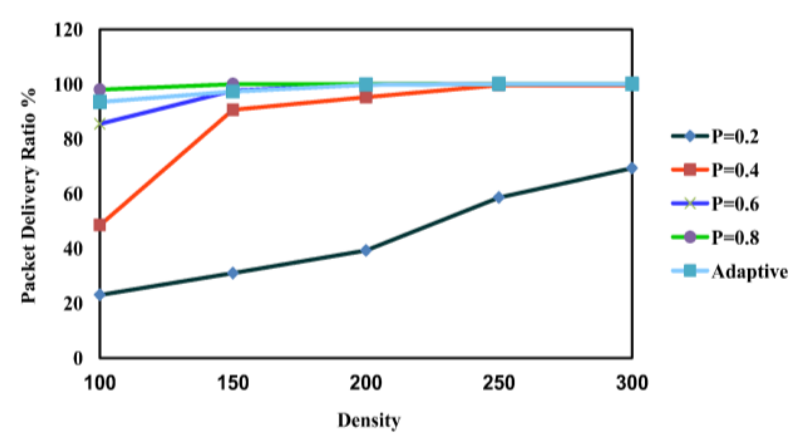

Figure 8 - Impact of density: PDR.

Fig. 9 presents the energy expended during the transmission and reception of messages of all nodes during simulation time, it shows that our algorithm has good results in terms of energy consumption for the different categories of network density; this improvement in the amount of energy consumed is proportional to an optimum reception rate. As we cited before, we have decreased the number of transmissions and receptions by using the fixed probabilistic-based flooding scheme. In this case, for a dense network, the probability threshold must be low in order to achieve an acceptable reception rate and, in the case of a sparse network, the probability threshold must be high. This technique imposes a fixed probability threshold for all nanonodes in the network regardless of their environmental traffic and conditions. The advantage of this approach is that it makes it possible to adjust the probability threshold for each nanonode independently of the other nodes by using its own environment conditions, consequently it reduces the number of transmissions for each nanonode basing on the traffic of its surroundings environment, and as a result it helps to conserve local energy.

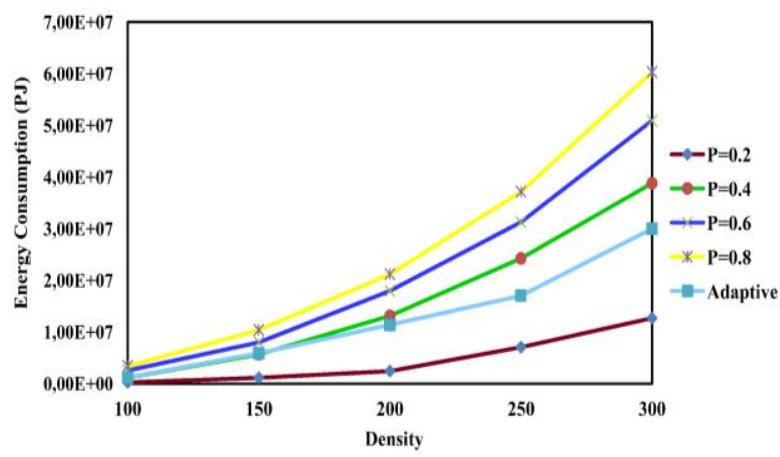

Figure 9 - Density vs Energy consumption
Fig. 10 depicts the comportment of latency for our adaptive approach compared with the fixed probabilistic-based flooding scheme, it shows the variation of the average latency as a function of the number of neighbors (density). The average delay is obtained by dividing the global sum of all forwarded packets delays by overall number of packets transmitted. The simulations indicate that in the usage of both approaches, the mean delay is basically the same in two cases: dense network and sparse network (for our experiments, the average latency reach 75 nanoseconds). If the threshold probability is 0.2 , the network will be completely disconnected because the average latency will be very low, but the packet delivery ratio will be very low.

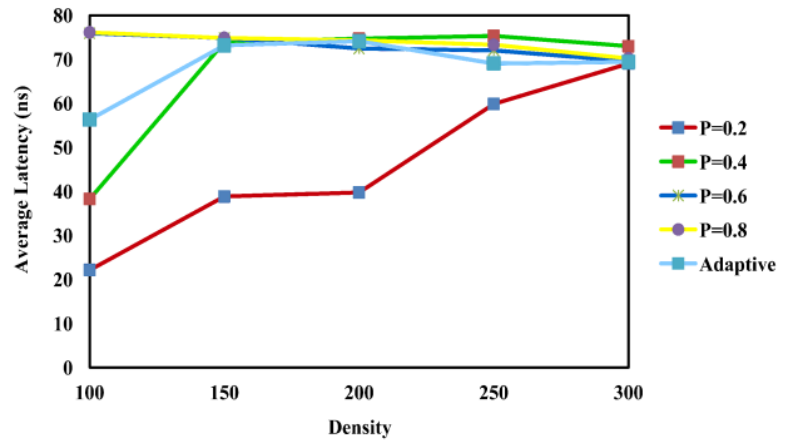

Figure 10 - Impact of density: Average latency

\section{CONCLUSION}

This paper has proposed an adaptive probabilistic-based broadcast algorithm for wireless nanosensor networks (WNSNs) to improve the data transmission success rate and energy consumption. The mechanism adjusts the rebroadcast probability by considering the number of neighbors for each nanonode. In order to prevent the excessive rebroadcasts, the rebroadcast probability of low density nanonodes is expanded while this one of high density nanonodes is reduced. Compared with the simple flooding and fixed probabilistic-based flooding, our simulations have confirmed, through analyses and simulations, the performance of these improvements with an important reduction of the excessive rebroadcasting packets. The aggregate of the probabilistic and the neighborhood learning gives good results in term of packet delivery ratio and energy consumption, even under conditions of excessive mobility and density. As an extension of this work in the future, we arrange firstly to combine our algorithm with a counter-based approach and note in case a combined execution enhancement is attainable. Secondly, we tend to propose an extremely adjusted probabilistic-based broadcast mechanism to facilitate the implementation of the 
best adaptation procedure using a game theoretical approach.

\section{REFERENCES}

[1] NS3 simulator. [Online]. Available at: https://www.nsnam.org. Accessed 13 Feb 2020.

[2] F. Al-Turjman, "A rational data delivery framework for disaster-inspired internet of nano-things (IONT) in practice," Cluster Computing, vol. 22, issue 1, pp. 1751-1763, 2019.

[3] I.F. Akyildiz, F. Brunetti, C. Blazquez, "Nanonetworks: A new communication paradigm. computer networks," The International Journal of Computer and Telecommunications Networking, vol. 52, pp. 2260-2279, 2008.

[4] I. Akyildiz, J. Jornet, "The internet of nanothings," Proceedings of the IEEE Conference on Wireless Communications, 2010, pp. 58-63.

[5] B. Atakan, S. Galmes, O.B. Akan, "Nanoscale communication with molecular arrays in nanonetworks," IEEE Transactions on NanoBioscience, vol. 11, pp. 149-160, 2012.

[6] Nano-sim. [Online]. Available at: http://telematics.poliba.it/. Accessed 05 Feb 2020.

[7] G. Piro, L.A. Grieco, G. Boggia, P. Camarda, "Nano-sim: Simulating electromagnetic-based nanonetworks in the network simulator 3," Proceedings of the 6th International ICST Conference on Simulation Tools and Techniques, Brussels, 2013, pp. 203-210.

[8] Z. W. J. Xu, R. Zhang, An energy efficient multi-hop routing protocol for terahertz wireless nanosensor networks, Proceedings of the International Conference on Wireless Algorithms, Systems, and Applications, 2016, pp. 367-376.

[9] J. Jornet, I. Akyilidiz, "Information capacity of pulse-based wireless nanosensor networks," Proceedings of the IEEE Conference on Sensor, Mesh and Ad Hoc Communications and Networks, SECON, 2011, pp. 80-88.

[10] J. Jornet, I. Akyilidiz, "Low-weight channel coding for interference mitigation in electromagnetic nanonetworks in the terahertz band," Proceedings of the IEE International Conference Communication (ICC), 2011, pp. $1-6$.

[11] J.M. Jornet, I.F. Akyildiz, "Graphene-based plasmonic nano-antenna for terahertz band communication in nanonetworks," IEEE Journal on Selected Areas in Communications, vol. 31, pp. 685-694, 2013.
[12] D. Kouvatsos, I. Mkwawa, "Broadcasting methods in mobile ad hoc networks: an overview," in: Kouvatsos D., editor, Proceedings of the HetNet, UK, Lecture Notes in Computer Science, vol. 5233, SpringerVerlag Berlin Heidelberg, 2011, pp. 767-83.

[13] C. Liaskos, A. Tsioliaridou, S. Ioannidis, N. Kantartzis, \& A. Pitsillides, "A deployable routing system for nanonetworks," Proceedings of the 2016 IEEE International Conference on Communications (ICC), 2016, pp. 1-6.

[14] F. Afsana, M. Asif-Ur-Rahman, M. R. Ahmed, M. Mahmud, M. S. Kaiser, An energy conserving routing scheme for wireless body sensor nanonetwork communication, IEEE Access, vol. 6, pp. 9186-9200, 2018.

[15] A. Oukhatar, M. Bakhouya, D. El Ouadghiri, K. Zine-Dine, "A performance evaluation of broadcasting algorithms in wireless nanosensor networks," Proceedings of the 2016 5th IEEE International Conference on Multimedia Computing and Systems (ICMCS), 2016, pp. 616-620.

[16] A. Oukhatar, M. Bakhouya, D. El Ouadghiri, K. Zine-Dine, "Probabilistic-based broadcasting for em-based wireless nanosensor networks," Proceedings of the 15th ACM International Conference on Advances in Mobile Computing \& Multimedia, New York, USA. 2017, pp. 232-236.

[17] M. Bakhouya, J. Gaber, P. Lorenz, "Energy evaluation of AID protocol in mobile ad hoc networks," Journal of Network and Computer Applications, vol. 58, pp. 287-293, 2015.

[18] A. Tsioliaridou, C. Liaskos, L. Pachis, S. Ioannidis, A. Pitsillides, "N3: Addressing and routing in 3d nanonetworks," Proceedings of the 2016 23rd International Conference on Telecommunications (ICT), 2016, pp. 1-6.

[19] A. Tsioliaridou, C. Liaskos, S. Ioannidis, A. Pitsillides, "Corona: A coordinate and routing system for nanonetworks," Proceedings of the Second ACM Annual International Conference on Nanoscale Computing and Communication, 2015, pp. 1-18.

[20] Y.-C. Tseng, S.-Y. Ni, Y.-S. Chen, \& J.-P. Sheu, "The broadcast storm problem in a mobile ad hoc network," Wireless Networks, vol. 8, issues 2/3, pp. 153-167, 2002.

[21] H. Yu, B. Ng, W. K. G. Seah, \& Y. Qu, "TTLbased efficient forwarding for the backhaul tier in nanonetworks," Proceedings of the 2017 14th IEEE Annual Consumer Communications \& Networking Conference (CCNC), 2017, pp. 554-559. 
[22] S.R. Neupane, "Routing in resource constrained sensor nanonetworks," Appl. Soft Comput., vol. 26, pp. 285-298, 2014.

[23] S. Sharma, D. Bhatia, "Static cluster PBDA localization algorithm for wireless nanosensor networks in terahertz communication band," in: Mathur G., Sharma H., Bundele M., Dey N., Paprzycki M. (eds), International Conference on Artificial Intelligence: Advances and Applications 2019. Algorithms for Intelligent Systems, Springer, Singapore, 2020, pp. 303310.

[24] M. Pierobon, "NASAIA. A routing framework for energy harvesting wireless nanosensor networks in the terahertz band," Wirel. Netw., vol. 20, issue 5, pp. 1169-1183, 2014.

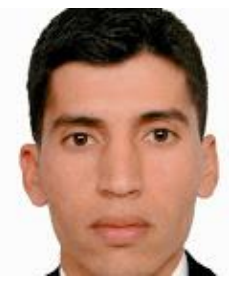

Ayoub Oukhatar, received engineer degree in Telecommunications and Networks from National School of Applied Sciences of Tetuan in 2014, Abdelmalek Essadi University. $\mathrm{He}$ is currently Ph.D student at the Department of computer sciences at Faculty of sciences of Méknes, Moulay Isamail University, Morocco. His research interests include wireless sensor networks, channel and network coding.

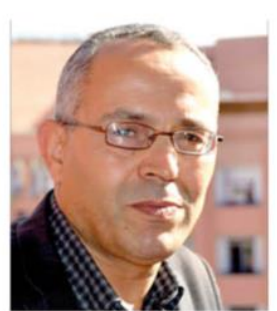

Driss El Ouadghiri is a full professor at the Science Faculty in Moulay Ismail University, Meknes, Morocco. He is also leader of Computer Science and Applications Laboratory and of Advanced Technologies and Networks research team. He was born in Ouarzazate, Morocco. In 2000 he got his $P h D$ in performance evaluation in wide area networks from Moulay Ismail University, Meknes, Morocco. His research interests focus on evaluation performance in networks (modelling and simulation),

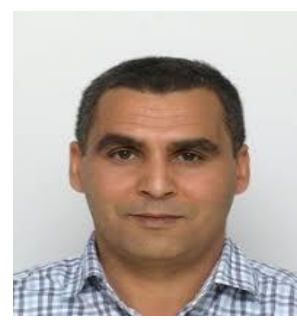

Mohamed Bakhouya is an associate professor at International University of Rabat. He obtained his HDR from UHA-France in 2013 and his PhD from UTBM-France in 2005. He has more than five years experiences in participa-

ting and working in sponsored ICT projects. He was $\mathrm{PI}$ of a starting grant at Aalto University-Finland (2011-2013), Co-PI (UTBM side) of two European projects ASSET (Advanced Safety and Driver Support in Efficient Road Transport, FP7-SST, 2008-2011, and TELEFOT (Field Operational Tests of Aftermarket and Nomadic Devises in Vehicles, FP7-ICT, 2008-2012. 